TRANSACTIONS OF THE

AMERICAN MATHEMATICAL SOCIETY

Volume 358, Number 11, November 2006, Pages 4821-4841

S 0002-9947(06)04145-6

Article electronically published on April 11, 2006

\title{
STATIONARY ISOTHERMIC SURFACES AND UNIFORMLY DENSE DOMAINS
}

\author{
R. MAGNANINI, J. PRAJAPAT, AND S. SAKAGUCHI
}

\begin{abstract}
We establish a relationship between stationary isothermic surfaces and uniformly dense domains. A stationary isothermic surface is a level surface of temperature which does not evolve with time. A domain $\Omega$ in the $N$ dimensional Euclidean space $\mathbb{R}^{N}$ is said to be uniformly dense in a surface $\Gamma \subset$ $\mathbb{R}^{N}$ of codimension 1 if, for every small $r>0$, the volume of the intersection of $\Omega$ with a ball of radius $r$ and center $x$ does not depend on $x$ for $x \in \Gamma$.

We prove that the boundary of every uniformly dense domain which is bounded (or whose complement is bounded) must be a sphere. We then examine a uniformly dense domain with unbounded boundary $\partial \Omega$, and we show that the principal curvatures of $\partial \Omega$ satisfy certain identities.

The case in which the surface $\Gamma$ coincides with $\partial \Omega$ is particularly interesting. In fact, we show that, if the boundary of a uniformly dense domain is connected, then (i) if $N=2$, it must be either a circle or a straight line and (ii) if $N=3$, it must be either a sphere, a spherical cylinder or a minimal surface. We conclude with a discussion on uniformly dense domains whose boundary is a minimal surface.
\end{abstract}

\section{INTRODUCTION}

The study of the evolution of isothermic sub-manifolds in a thermic conductor is fascinating. In a typical situation, we have a thermic conductor which, initially, is homogeneously warm (constant positive temperature) and whose boundary is constantly kept at zero temperature. As time goes by, the conductor cools down and, depending on its shape, its hot spots (points of relatively maximal temperature) and isothermic surfaces (surfaces where temperature is constant) have various evolutions.

In certain instances, hot spots or isothermic surfaces do not evolve - we say that they are stationary; for example, in a spherical conductor, the (unique) hot spot does not move with time and, at each time, isothermic surfaces are all spherical.

We are interested in characterizing conductors with stationary isothermic submanifolds. While this task is very hard for the case of stationary hot spots (see [K1], [CS], GW], [Kw, [MS2, [MS4, for some partial and related results on this issue), the study of conductors with stationary isothermic surfaces has produced more satisfactory results. (After L. Zalcman [Z, we usually refer to this problem

Received by the editors August 11, 2004.

2000 Mathematics Subject Classification. Primary 35K05, 35K20; Secondary 53A10, 58J70.

Key words and phrases. Stationary surfaces, uniformly dense domains, minimal surfaces.

This research was partially supported by a Grant-in-Aid for Scientific Research (B) ( $\sharp 12440042$ ) and $(B)(\sharp 15340047)$ of the Japan Society for the Promotion of Science and by a Grant of the Italian MURST.

(C)2006 American Mathematical Society Reverts to public domain 28 years from publication 
as the Matzoh Ball Soup.) The first one is due to G. Alessandrini, who proved in $\mathrm{As}$, that the ball is the only bounded conductor with the property that all its isothermic surfaces are stationary. In [MS1, it is shown that one arrives at the same conclusion even if the conductor contains only one fairly regular isothermic surface.

If the conductor is not bounded, other solutions are possible. One can easily show that, in the case of a half space or a solid circular cylinder, all isothermic surfaces are always hyperplanes or circular cylinders, respectively. However, the problem of classifying conductors with a stationary isothermic surface is still open. We refer the interested reader to the reference [MS3 for some partial results in this direction.

In this paper, we consider a slightly different physical situation: instead of a conductor, we have an infinite conducting wall divided into two regions; one is initially (homogeneously) warm while the other is cold. In mathematical terms, we have in mind the following initial value problem for the heat equation:

$$
\begin{array}{ll}
u_{t}=\Delta u & \text { in } \mathbb{R}^{N} \times(0,+\infty), \\
u=\mathcal{X}_{\Omega} & \text { on } \mathbb{R}^{N} \times\{0\},
\end{array}
$$

where $\mathcal{X}_{\Omega}$ is the characteristic function of a domain $\Omega$ in the $N$-dimensional Euclidean space $\mathbb{R}^{N}, N \geq 2$. We want to classify all the domains $\Omega$ such that there is an $(N-1)$-dimensional surface $\Gamma \subset \mathbb{R}^{N}$ which is a stationary isothermic surface, that is, such that at each time $t>0$, the temperature $u=u(x, t)$ remains constant on $\Gamma$ (a constant depending on $t$ ). This problem is slightly easier than the one considered in MS1, and, besides producing surprising results, it may be useful to build up more insight on the Matzoh Ball Soup problem.

We observe in passing that, in a well-known paper [CK], I. Chavel and L. Karp have shown that, if $\Omega$ is bounded and the solution of (1.1)-(1.2) has a stationary level surface $\Gamma$, then $\Gamma$ must be a sphere (see [CK, Theorem 2, p. 275]). In Theorem 3.1 we will give an alternative and more direct proof of this result, under slightly more general assumptions. It is clear that the same conclusion can be drawn when $\Omega$ is the complement of a bounded domain.

When $\partial \Omega$ is also unbounded, our problem should be studied by different means. To this aim, in Theorem 1.1 below, we establish a relationship between stationary isothermic surfaces and what we call uniformly dense domains.

Let $B(x, r)$ be the open ball of positive radius $r$ and center $x \in \mathbb{R}^{N}$. If $x \in \mathbb{R}^{N}$ and $r>0$, we define the (spherical) average $r$-density of $\Omega$ at $x$ as the ratio

$$
\rho(x, r)=\frac{|\Omega \cap B(x, r)|}{|B(x, r)|} .
$$

(We shall use the same symbol - single bars - to denote both the $N$-dimensional Lebesgue measure and the $(N-1)$-dimensional Hausdorff measure of sets; thus, for instance, $|\Omega|$ and $|\partial \Omega|$ indicate the $N$-Lebesgue measure of $\Omega$ and the $(N-1)$ Hausdorff measure of $\partial \Omega$, respectively. We will use the notation $\omega_{N}=|\partial B(0,1)|$.) If $\Gamma \subset \mathbb{R}^{N}$, we say that $\Omega$ is uniformly dense in $\Gamma$ if it satisfies the following property:

(1.4) there exists $r_{0} \in(\operatorname{dist}(\Gamma, \partial \Omega),+\infty]$ such that, for each fixed $r \in\left(0, r_{0}\right)$, the function $x \mapsto \rho(x, r)$ is constant on $\Gamma$. 
Theorem 1.1. Let $\Omega$ be a domain in $\mathbb{R}^{N}$ and let $u$ be the solution of (1.1)-(1.2). Then $\Gamma \subset \mathbb{R}^{N}$ is a stationary isothermic surface for $u$ if and only if $\Omega$ is uniformly dense in $\Gamma$ with $r_{0}=+\infty$.

Thus, our problem is reduced to the study of uniformly dense domains. It is clear that, if $\Omega$ is uniformly dense in $\Gamma$, then any $x \in \Gamma$ must have the same distance from $\partial \Omega$. In other words, $\Gamma$ must be parallel to a portion of $\partial \Omega$ and, for this reason, many of the properties of $\partial \Omega$ will be inherited by $\Gamma$. In the remainder of this section, we shall discuss the case in which $\Gamma$ is (a portion of) $\partial \Omega$, referring the reader to Theorem 3.6, where the general case is carried out.

A first important remark is that property (1.4) implies smoothness. In fact, in Theorem 2.2, we shall prove that, if (a portion of) the boundary of a uniformly dense domain is locally the graph of a continuous function (from now on, we shall say that $\partial \Omega$ is of class $C^{0}$ ), then it must be smooth (and even analytic when $r_{0}$ can be chosen to be infinite).

A more important result is the following theorem.

Theorem 1.2. Let $\Omega$ be a domain in $\mathbb{R}^{N}, N \geq 2$, and suppose $\Gamma$ is an open subset of $\partial \Omega$ of class $C^{0}$.

If $\Omega$ is uniformly dense in $\Gamma$ (with $r_{0}$ either finite or infinite), then the mean curvature of $\Gamma$ is constant. In particular, if $\rho(x, r)=1 / 2$ for every $x \in \Gamma$ and every sufficiently small $r>0$, then $\Gamma$ must have zero mean curvature.

As a consequence of this statement, when $\Gamma=\partial \Omega$ and $\partial \Omega$ is bounded, by Aleksandrov's Soap Bubble Theorem (see [A] ), we can infer that $\partial \Omega$ must be a sphere. We incidentally observe that this symmetry result has an interesting generalization: let $a=\left(a_{1}, \ldots, a_{N}\right)$ be a vector with positive entries and let $E_{a}(0,1)$ be an ellipsoid centered at 0 and with semi-axes $a_{1}, \ldots, a_{N}$; if (1.4) holds for $\Gamma=\partial \Omega$ and with the balls $B(x, r)$ replaced by the ellipsoids $E_{a}(x, r)=x+r E_{a}(0,1)$, then, modulo a translation, $\Omega$ must be an ellipsoid homothetic to $E_{a}(0,1)$ as well (see Corollary 3.4).

If $\Omega$ is a planar domain, by Theorem 1.2 we can conclude that a uniformly dense domain in $\partial \Omega$ is such that any connected component of $\partial \Omega$ is either a circle or a straight line. We note here that this result can also be obtained by a simple and purely geometric argument. In fact, if the curvature $\kappa$ of $\partial \Omega$ is not constant, we can find two points $x, \xi \in \partial \Omega$ such that $\kappa(x)<\kappa(\xi)$; if $r>0$ is chosen sufficiently small, we can prove that $|\Omega \cap B(x, r)|<|\Omega \cap B(\xi, r)|$, thus contradicting property (1.4). Indeed, if $\kappa(x)<\kappa(\xi)$, we can choose a suitable rigid motion $\mathcal{R}$ (a translation plus a rotation) that moves $x$ to $\xi$, the tangent line to $\partial \Omega$ at $x$ to the tangent line to $\partial \Omega$ at $\xi$, and such that

$$
\mathcal{R}(\Omega \cap B(x, r)) \subset \Omega \cap B(\xi, r) \text { and }|(\Omega \cap B(\xi, r)) \backslash \mathcal{R}(\Omega \cap B(x, r))|>0,
$$

so that $|\Omega \cap B(x, r)|=|\mathcal{R}(\Omega \cap B(x, r))|<|\Omega \cap B(\xi, r)|$.

In Theorem 1.3 below, we present our results for the case $N \geq 3$. (In what follows, $K_{j}(x), j=1, \ldots, N-1$, denote the $j$-th symmetric invariant $K_{j}(x)$ of the surface $\partial \Omega$ evaluated at $x \in \partial \Omega$; see Section 3 for the definition.)

Theorem 1.3. Let $\Omega$ be a domain in $\mathbb{R}^{N}, N \geq 3$, and suppose $\Gamma$ is an open subset of the boundary $\partial \Omega$ of class $C^{0}$.

If $\Omega$ is uniformly dense in $\Gamma$, then

(i) if $N=3, K_{1}^{3}-4 K_{1} K_{2}$ is constant in $\Gamma$; 
(ii) if $N \geq 4, K_{1}^{3}-4 K_{1} K_{2}+4 K_{3}$ is constant in $\Gamma$.

In particular, if $N=3$, we conclude that $\Gamma$ must be either a portion of a sphere, of a spherical cylinder or of a minimal surface.

A plane is a trivial example of a minimal surface which is the boundary of a uniformly dense domain. A non-trivial example does exist, though: a right helicoid $\mathcal{H}$ is indeed a minimal surface which splits the space $\mathbb{R}^{3}$ up into two uniformly dense domains, and we have that $\rho(x, r)=1 / 2$ for every $x \in \partial \Omega$ and every positive $r$. Moreover, we note that the symmetry of $\mathcal{H}$ implies that the solution $u$ of (1.1)(1.2) satisfies $u=\frac{1}{2}$ on $\mathcal{H} \times(0,+\infty)$. It is evident that, in general dimension, the hypersurface $\partial \Omega=\mathcal{H} \times \mathbb{R}^{N-3}$ will have the same property. W. Reichel (University of Basel) brought to our attention a paper by J. Nitsche $[\mathrm{N}$, where it is proven that the plane and the right helicoid are the only (minimal) surfaces in $\mathbb{R}^{3}$ such that $\rho \equiv 1 / 2$. Both Nitsche's result $[\mathrm{N}]$ and Theorem 1.3 above are derived by computing the Taylor's formula for $\rho(x, r)$ near $r=0$ up to the relevant degrees. In Section 5. we report our computations for such a formula in general dimension, since they are needed to prove Theorem 1.3 .

Nitsche's result does not rule out the existence of minimal surfaces (other than the helicoid or the plane) which are boundaries of uniformly dense domains. Here, we consider the case of embedded minimal surfaces of finite total curvature. The theory of complete embedded minimal surfaces of finite total curvature in $\mathbb{R}^{3}$ has developed recently (see [HK], [LM], and [PR] for some surveys). In particular, in $\mathrm{Kp}$ N. Kapouleas constructed large families of such minimal surfaces with symmetries, and moreover in T] M. Traizet showed the existence of such minimal surfaces with no symmetries. Note that the catenoid and the plane are the classical examples of complete embedded minimal surfaces of finite total curvature, and the helicoid is not of finite total curvature because of its periodicity.

By combining Nitsche's result [N] and the theory of complete embedded minimal surfaces of finite total curvature in $\mathbb{R}^{3}$, we conclude our analysis of uniformly dense domains with the following result.

Theorem 1.4. Let $S$ be a complete embedded minimal surface of finite total curvature in $\mathbb{R}^{3}$, and let $\Omega$ be one of the two domains disconnected by $S$ from $\mathbb{R}^{3}$.

If $\Omega$ is uniformly dense in $S(=\partial \Omega)$, then $S$ must be a plane.

\section{REgularity of STATIONARY ISOTHERMiC SURFACES AND UNIFORMLY DENSE DOMAINS}

Theorem 1.1 establishes the connection between stationary isothermic surfaces and uniformly dense domains. In order to prove it, we note that (1.4) holds if and only if

$$
\text { there exists } r_{0} \in(\operatorname{dist}(\Gamma, \partial \Omega),+\infty] \text { such that, }
$$

for almost every fixed $r \in\left(0, r_{0}\right)$, the function $x \mapsto \sigma(x, r)$ is constant on $\Gamma$,

where

$$
\sigma(x, r)=\frac{|\Omega \cap \partial B(x, r)|}{|\partial B(x, r)|} .
$$

Proof of Theorem 1.1. The solution of problem (1.1)-(1.2) is

$$
u(x, t)=(4 \pi t)^{-\frac{N}{2}} \int_{\mathbb{R}^{N}} \mathcal{X}_{\Omega}(\xi) e^{-\frac{|x-\xi|^{2}}{4 t}} d \xi, x \in \mathbb{R}^{N}, t>0 .
$$


We compute that

$$
\begin{aligned}
u(x, t) & =(4 \pi t)^{-\frac{N}{2}} \int_{\mathbb{R}^{N}} \mathcal{X}_{\Omega}(\xi) e^{-\frac{|x-\xi|^{2}}{4 t}} d \xi \\
& =(4 \pi t)^{-\frac{N}{2}} \int_{0}^{+\infty} e^{-\frac{r^{2}}{4 t}}\left(\int_{\partial B(x, r)} \mathcal{X}_{\Omega}(\xi) d S_{\xi}\right) d r \\
& =(4 \pi t)^{-\frac{N}{2}} \int_{0}^{+\infty} e^{-\frac{r^{2}}{4 t}}|\Omega \cap \partial B(x, r)| d r .
\end{aligned}
$$

Let $p, q \in \Gamma$ be any pair of points. Since the Laplace transform is injective, (2.4) implies that $u(p, t)=u(q, t)$ for every $t>0$ if and only if $|\Omega \cap \partial B(p, r)|=$ $|\Omega \cap \partial B(q, r)|$ for almost every $r>0$. This completes the proof.

Theorem 2.1 below is an analog of [MS1, Lemma 3.1].

Theorem 2.1. Let $\Omega$ be a domain in $\mathbb{R}^{N}, N \geq 2$, satisfying the interior sphere condition, and suppose that $D$ is a domain satisfying the interior cone condition and such that $\bar{D} \subset \mathbb{R}^{N} \backslash \bar{\Omega}$.

If $\Gamma=\partial D$ is a stationary isothermic surface for the solution $u$ of (1.1)-(1.2), then there exists an open subset $\Lambda$ of $\partial \Omega$ satisfying the following properties:

(i) both $\Gamma$ and $\Lambda$ are analytic;

(ii) $\Gamma$ and $\Lambda$ are parallel;

(iii) each principal curvature of $\Lambda$ is smaller than the number $\frac{1}{R}$, where $R=$ $\operatorname{dist}(\Gamma, \partial \Omega)$.

Proof. We can use Theorem 2.5 below. The analyticity of $\Gamma$ follows from the analyticity of $u$ in $x$.

When the stationary isothermic surface $\Gamma$ coincides with $\partial \Omega$, we can substantially weaken the assumptions of Theorem 2.1.

Theorem 2.2. Let $\Omega$ be an open set in $\mathbb{R}^{N}$ with boundary $\partial \Omega$ of class $C^{0}$.

If $\Gamma=\partial \Omega$ is a stationary isothermic surface for the solution $u$ of (1.1)-(1.2), then $\Gamma$ is analytic.

Proof. By Theorem $1.1 \Omega$ is uniformly dense if and only if $\partial \Omega$ is a stationary level surface of the function $u$ defined by (2.3).

It suffices to show that, for every point $x \in \partial \Omega$, there exists a time $t_{0}>0$ such that $\nabla u\left(x, t_{0}\right) \neq 0$; then, analyticity of $\partial \Omega$ will follow from analyticity of $u$ with respect to the space variable.

Assume, by contradiction, that there exists a point $x_{0} \in \partial \Omega$ such that $\nabla u\left(x_{0}, t\right)=$ 0 for every $t>0$. Then, by Corollary 2.2 in MS1, we have that

$$
\int_{B\left(x_{0}, r\right)}\left(\xi-x_{0}\right) u(\xi, t) d \xi=0,
$$

for every positive $t$ and $r$. By letting $t$ tend to zero, we then obtain that

$$
\int_{\Omega \cap B\left(x_{0}, r\right)}\left(\xi-x_{0}\right) d \xi=0,
$$

for every $r>0$. Hence, $x_{0}$ must be the center of mass of every set $\Omega \cap B\left(x_{0}, r\right)$. 
Now, by choosing $r>0$ sufficiently small and by eventually translating and rotating the axes, we can suppose that $x_{0}=0$ and that $\partial \Omega$ be represented, in a neighborhood of $x_{0}=0$, by the graph of a continuous function $\varphi: U(0) \rightarrow \mathbb{R}$, where $U(0) \subset \mathbb{R}^{N-1}$ is a suitable neighborhood of 0 and $\varphi(0)=0$.

Let $\varphi_{ \pm}(y)=\max \left[\varphi(y), \pm \sqrt{r^{2}-|y|^{2}}\right]$ for $y \in B^{\prime}=\left\{y \in \mathbb{R}^{N-1}:|y|<r\right\} \subset U(0)$; the set $\Omega \cap B(x, r)$ can be represented as $\left\{\left(y, y_{N}\right) \in B^{\prime} \times \mathbb{R}: \varphi_{-}(y)<y_{N}<\varphi_{+}(y)\right\}$. Therefore, we can infer that

$$
\begin{aligned}
& \int_{\Omega \cap B(x, r)}\left(\xi_{N}-x_{N}\right) d \xi=\int_{\Omega \cap B(x, r)} y_{N} d y_{N} d y \\
& =\int_{B^{\prime}}\left(\int_{\varphi_{-}(y)}^{\varphi_{+}(y)} y_{N} d y_{N}\right) d y=\frac{1}{2} \int_{B^{\prime}}\left[\varphi_{+}(y)^{2}-\varphi_{-}(y)^{2}\right] d y>0 .
\end{aligned}
$$

This contradicts (2.5)).

Theorems 1.1, 2.1 and 2.2 yield the following regularity result for uniformly dense domains.

Corollary 2.3. Let $\Omega$ be an open set in $\mathbb{R}^{N}$ and suppose that $\Gamma$ satisfies the assumption of either Theorem 2.1 or 2.2 .

If $\Omega$ is uniformly dense in $\Gamma$ with $r_{0}=+\infty$, then $\Gamma$ is analytic.

In case $r_{0}$ is finite, we prove the following results.

Theorem 2.4. Let $\Omega$ be an open set in $\mathbb{R}^{N}$ with boundary $\partial \Omega$ of class $C^{0}$, and let $\Gamma$ be an open subset of $\partial \Omega$.

If $\Omega$ is uniformly dense in $\Gamma$, then $\Gamma$ must be smooth.

Proof. Choose $\psi \in C_{0}^{\infty}\left(\mathbb{R}^{N}\right)$ such that supp $(\psi) \subset B\left(0, r_{0}\right)$ and $\psi(x)=\eta(|x|)$. The convolution $\psi * \mathcal{X}_{\Omega}$ belongs to $C^{\infty}\left(\mathbb{R}^{N}\right)$, and we have that

$$
\begin{aligned}
\psi * \mathcal{X}_{\Omega}(x) & =\int_{B\left(0, r_{0}\right)} \psi(\xi) \mathcal{X}_{\Omega}(x-\xi) d \xi \\
& =\int_{0}^{r_{0}} \eta(r)\left(\int_{\Omega \cap \partial B(x, r)} d S_{\xi}\right) d r .
\end{aligned}
$$

Since $\Omega$ satisfies (2.1) we infer that $\psi * \mathcal{X}_{\Omega}$ must be constant on $\Gamma$ and hence $\Gamma$ is the level surface of a smooth function. If we prove that, for every $x \in \Gamma$ there is a smooth function $\psi$ such that $\psi * \mathcal{X}_{\Omega}$ has non-vanishing gradient at $x$, then, by the implicit function theorem, we can conclude that $\Gamma$ is smooth.

Similar to the proof of Theorem 2.2, we will show that, if there exists a point $x_{0} \in \Gamma$ such that $\nabla\left(\psi * \mathcal{X}_{\Omega}\right)\left(x_{0}\right)=0$ for every function $\psi$ with the properties stated above, then (2.5) must hold for every $r \in\left(0, r_{0}\right)$, thus getting a contradiction.

Since

$$
\begin{aligned}
\nabla\left(\psi * \mathcal{X}_{\Omega}\right)\left(x_{0}\right) & =\int_{B\left(x_{0}, r_{0}\right)} \mathcal{X}_{\Omega}(\xi) \eta^{\prime}\left(\left|x_{0}-\xi\right|\right) \frac{x_{0}-\xi}{\left|x_{0}-\xi\right|} d \xi \\
& =\int_{0}^{r_{0}} \eta^{\prime}(r)\left(\int_{\Omega \cap \partial B\left(x_{0}, r\right)} \frac{x_{0}-\xi}{\left|x_{0}-\xi\right|} d S_{\xi}\right) d r
\end{aligned}
$$


then

$$
\int_{0}^{r_{0}} \eta^{\prime}(r) M(r) d r=0
$$

where

$$
M(r)=\int_{\Omega \cap \partial B\left(x_{0}, r\right)} \frac{x_{0}-\xi}{\left|x_{0}-\xi\right|} d S_{\xi}
$$

Equation (2.6) implies that the distributional derivative of the bounded function $M(r)$ equals zero on $\left(0, r_{0}\right)$. Therefore, by observing that

$$
\lim _{r \rightarrow 0^{+}} M(r)=0
$$

we conclude that $M(r)$ equals zero for almost every $r \in\left(0, r_{0}\right)$, and (2.5) holds for every $r \in\left(0, r_{0}\right)$.

Theorem 2.5. Let $\Omega$ be an open set in $\mathbb{R}^{N}$ satisfying the interior sphere condition, and suppose that $D$ is a domain satisfying the interior cone condition and such that $\bar{D} \subset \mathbb{R}^{N} \backslash \bar{\Omega}$. Let $\Gamma=\partial D$.

If $\Omega$ is uniformly dense in $\Gamma$, then there exists an open subset $\Lambda$ of $\partial \Omega$ satisfying the following properties:

(i) both $\Gamma$ and $\Lambda$ are smooth;

(ii) $\Gamma$ and $\Lambda$ are parallel;

(iii) each principal curvature of $\Lambda$ is smaller than the number $\frac{1}{R}$, where $R=$ $\operatorname{dist}(\Gamma, \partial \Omega)$.

Remark 2.6. We stress the fact that item (iii) will be essential for the proof of Theorem 5.5: it is important that each principal curvature of $\Lambda$ never equals $\frac{1}{R}$.

As a matter of fact, it is not difficult to prove that each principal curvature of $\Lambda$ is not greater than $\frac{1}{R}$. Indeed, for every $x \in \Gamma$, there exists a unique point $y \in \Lambda$ such that $\overline{B(x, R)} \cap \bar{\Omega}=\{y\}$ and hence, at the point $y \in \Lambda$, the two surfaces $\Lambda$ and $\partial B(x, R)$ are tangent to each other. Once we have chosen the normal vector at $y \in \Lambda$ for both surfaces in such a way that the principal curvatures of $\partial B(x, R)$ equal $\frac{1}{R}$ (note that each principal curvature changes its sign if one chooses the normal vector in the opposite direction), we obtain the desired conclusion.

Proof of Theorem 2.5. We proceed as in the proof of Theorem 2.4 and calculate $\psi * \mathcal{X}_{\Omega}(x)$ and $\nabla\left(\psi * \mathcal{X}_{\Omega}\right)(x)$. By supposing that there exists a point $x_{0} \in \Gamma$ such that $\nabla\left(\psi * \mathcal{X}_{\Omega}\right)\left(x_{0}\right)=0$ for every function $\psi$ with the properties stated at the beginning of the proof of Theorem 2.4 we conclude that (2.5) holds for every $r \in\left(0, r_{0}\right)$. Define the function $d=d(x)$ by

$$
d(x)=\operatorname{dist}(x, \partial \Omega), \quad x \in \mathbb{R}^{N} \backslash \bar{\Omega} .
$$

Since $\Omega$ is uniformly dense in $\Gamma$, as is observed in the Introduction, we have

$$
d(x)=R \text { for every } x \in \Gamma,
$$

where $R=\operatorname{dist}(\Gamma, \partial \Omega)$. Since $D$ satisfies the interior cone condition, there exists a finite right spherical cone $K$ with vertex at $x_{0}$ such that $K \subset \bar{D}$ and $\bar{K} \cap \partial D=\left\{x_{0}\right\}$. By translating and rotating if needed, we can suppose that $x_{0}=0$ and that $K$ is the set $\left\{x \in B(0, \rho): x_{N}<-|x| \cos \theta\right\}$, for some choice of $\rho \in(0, R)$ and $\theta \in\left(0, \frac{\pi}{2}\right)$.

Since $K \subset \bar{D}$ and $\bar{K} \cap \partial D=\{0\}$, (2.8) implies that

$$
d(x)>R \text { for every } x \in K \text {. }
$$


The set defined by

$$
V=\left\{x \in \partial B(0, R): x_{N} \geq R \sin \theta\right\}
$$

is such that

$$
\partial \Omega \cap \partial B(0, R) \subset V,
$$

because, otherwise, there would be a point in $K$ contradicting (2.9). Thus, from (2.11) it follows that there exists a number $\delta>0$ such that

$$
d(x) \geq \delta \text { for every } x \in \partial B(0, R) \cap\left\{x_{N} \leq R \sin \frac{\theta}{2}\right\} .
$$

Choose $r \in\left(R, \min \left\{R+\delta, r_{0}\right\}\right)$. Then (2.12) yields that

$$
\Omega \cap B(0, r) \subset B(0, r) \cap\left\{x_{N} \geq R \sin \frac{\theta}{2}\right\} .
$$

This contradicts the fact that (2.5) holds for $x_{0}=0$.

Therefore, it follows that $\Gamma$ must be smooth, and we can complete the proof by following that of [MS1, Lemma 3.1].

\section{Symmetry of STATIONARY ISOTHERMiC SURFACES AND UNIFORMLY DENSE DOMAINS}

We begin with a simpler proof of a slightly more general version of Theorem 2 in $\mathrm{CK}$.

Theorem 3.1. Let $\Omega$ be any measurable set in $\mathbb{R}^{N}$ such that

$$
\text { either } \int_{\Omega}|\xi|^{2} d \xi<+\infty \quad \text { or } \quad \int_{\mathbb{R}^{N} \backslash \Omega}|\xi|^{2} d \xi<+\infty
$$

and let $u$ be given by (2.3). Assume that there exists a non-empty open surface $\Gamma \subset \mathbb{R}^{N}$ which is a stationary isothermic surface for $u$.

Then $\Gamma$ extends as a stationary isothermic surface for $u$ to a whole sphere centered at the point $x_{0}$, where $x_{0}$ equals

$$
\text { either } \frac{1}{|\Omega|} \int_{\Omega} \xi d \xi \quad \text { or } \quad \frac{1}{\left|\mathbb{R}^{N} \backslash \Omega\right|} \int_{\mathbb{R}^{N} \backslash \Omega} \xi d \xi,
$$

depending on (3.1).

Proof. First, we prove this theorem when $\int_{\Omega}|\xi|^{2} d \xi<+\infty$. The idea of the proof is to manipulate the expression (2.3) for $u$ in order to obtain the $L^{p}$-norm of a suitable function for some suitable $p>0$.

In fact, from (2.3) we write

$$
\left\{(4 \pi t)^{N / 2}|\Omega|^{-1} u(x, t)\right\}^{4 t}=\left\|f_{x}\right\|_{L^{\frac{1}{4 t}}(\Omega, \mu)},
$$

where $f_{x}(\xi)=e^{-|x-\xi|^{2}}$ for $x, \xi \in \mathbb{R}^{N}$ and $\mu$ is the normalized Lebesgue measure such that $d \mu(\xi)=d \xi /|\Omega|$.

Since $\Gamma$ is a stationary isothermic surface for $u$, the left-hand side of (3.2) depends only on $t$ when $x \in \Gamma$. By taking the limit as $t \rightarrow+\infty$ in (3.2), we see that there exists a constant $c_{\infty}$ such that

$$
\exp \left\{\int_{\Omega} \log f_{x}(\xi) d \mu(\xi)\right\}=c_{\infty},
$$


which implies that

$$
\left|x-x_{0}\right|^{2}=\left|x_{0}\right|^{2}-\frac{1}{|\Omega|} \int_{\Omega}|\xi|^{2} d \xi-\log c_{\infty} \text { for } x \in \Gamma,
$$

that is, $\Gamma$ is contained in a sphere centered at $x_{0}$. In conclusion, the analyticity of $u$ yields that $\Gamma$ extends as a stationary isothermic surface for $u$ to the whole sphere.

When $\int_{\mathbb{R}^{N} \backslash \Omega}|\xi|^{2} d \xi<+\infty$, it suffices to replace $u$ by $1-u$ in the above argument.

Corollary 3.2. Let $\Omega$ be a domain in $\mathbb{R}^{N}$ satisfying (3.1), and suppose that $\partial \Omega$ is connected. Let $u$ be given by (2.3). Assume that there exists a non-empty open surface $\Gamma \subset \mathbb{R}^{N}$ which is a stationary isothermic surface for $u$.

Then $\Omega$ must be either a ball or the exterior of a ball.

In what follows, we shall prove our symmetry results for stationary isothermic surfaces when $\Omega$ does not satisfy any assumption other than having its boundary of class $C^{0}$. Thanks to Theorem 1.1, we can work with a uniformly dense domain $\Omega$. In fact, we will derive our results under the weaker assumption that $\Omega$ obeys (1.4) with finite $r_{0}$.

The proofs in the remainder of this section are based on a technical result, the Taylor expansion for $\rho(x, r)$ at either $r=0$ or $r=\operatorname{dist}(x, \bar{\Omega})$, which will be derived later in Section 5

As mentioned in the Introduction, for $j \in\{1, \ldots, N-1\}, K_{j}(x)$ denotes the $j$-th symmetric invariant $K_{j}(x)$ of the surface $\partial \Omega$ evaluated at $x$, that is,

$$
K_{j}(x)=\sum_{i_{1}<\cdots<i_{j}} \kappa_{i_{1}}(x) \cdots \kappa_{i_{j}}(x), \quad j=1, \ldots, N-1,
$$

where $\kappa_{j}(x), j=1, \ldots, N-1$, are the principal curvatures of $\partial \Omega$ at $x$ (see Ma and $\left[\underline{\mathrm{R}}\right.$ for details). With this definition, $H=K_{1} /(N-1)$ and $K=K_{N-1}$ are the mean and the Gauss curvature of $\partial \Omega$, respectively.

We begin by the simpler case in which $\Gamma=\partial \Omega$ and prove Theorem 1.2 .

Proof of Theorem 1.2. Since $\Omega$ is uniformly dense in $\Gamma$, by Theorem 2.4, $\Gamma$ is smooth. By Theorem [5.2, for the function $\rho(x, r)$ defined in (1.3) we have that

$$
\rho(x, r)=\frac{1}{2}+\frac{N}{N+1} \sigma_{1}(x) r+O\left(r^{2}\right) \text { as } r \rightarrow 0 .
$$

Since $\rho(x, r)$ does not depend on $x$ when $x \in \Gamma$, for $x, \xi \in \Gamma$, we have

$$
0=\rho(x, r)-\rho(\xi, r)=\frac{N}{N+1}\left[\sigma_{1}(x)-\sigma_{1}(\xi)\right] r+O\left(r^{2}\right) \text { as } r \rightarrow 0 .
$$

Dividing the right-hand side of this equation by $r$ and letting $r \rightarrow 0$ implies that $\sigma_{1}(x)=\sigma_{1}(\xi)$, that is, the function $\sigma_{1}$ is constant on $\Gamma$. From the first formula in (5.7) and Lemma 5.4 we have

$$
\sigma_{1}(x)=-\frac{1}{\omega_{N}} \sum_{|\alpha|=1} \frac{D^{2 \alpha} \varphi(0)}{(2 \alpha) !} \int_{\mathbb{S}^{N-2}} v^{2 \alpha} d S_{v}=-\frac{\omega_{N-1}}{2(N-1) \omega_{N}} \Delta \varphi(0) .
$$

Also, by a standard formula (see [GT], for instance), we have that for any $y$ in some neighborhood of the origin in $\mathbb{R}^{N-1}$,

$$
(N-1) H(y, \varphi(y))=-\operatorname{div}\left(\frac{\nabla \varphi(y)}{\sqrt{1+|\nabla \varphi(y)|^{2}}}\right) .
$$


By recalling that $\varphi(0)=0$ and $\nabla \varphi(0)=0$, we let $y=0$ to obtain

$$
(N-1) H(x)=(N-1) H(0,0)=-\Delta \varphi(0),
$$

and hence

$$
\sigma_{1}(x)=\frac{\omega_{N-1}}{2 \omega_{N}} H(x) .
$$

Therefore, we conclude that the mean curvature $H$ of $\Gamma$ is constant.

When $\rho(x, r)=1 / 2$ for every $x \in \Gamma$ and every sufficiently small $r>0$, we have that $\sigma_{1}(x)=0$ and hence $H(x)=0$ for $x \in \Gamma$.

Corollary 3.3. Let $\Omega$ be a domain in $\mathbb{R}^{N}, N \geq 2$, with boundary $\partial \Omega$ of class $C^{0}$. Assume that $\Omega$ is uniformly dense in $\partial \Omega$.

Then $\partial \Omega$ is analytic and

(i) if $\partial \Omega$ is compact, then it must be a sphere;

(ii) if $N=2$, then either any connected component of $\partial \Omega$ is a circle or a straight line.

Proof. Since $H$ equals a constant $H_{0}$ on $\partial \Omega$, then $\partial \Omega$ must be an analytic surface.

(i) By Aleksandrov's Soap Bubble Theorem (see [Al]), we infer that $\partial \Omega$ must be a sphere, since $\partial \Omega$ is compact.

(ii) If $N=2$, then either $H_{0} \neq 0$ or $H_{0}=0$, i.e. either any connected component of $\partial \Omega$ is a circle or a straight line.

Let $a \in \mathbb{R}^{N}$ be a vector with positive entries $a_{1}, \ldots, a_{N}$. In the following result, we will assume that $\left\{E_{a}(x, r)\right\}_{r>0}$ is a family of ellipsoids homothetic to a reference ellipsoid $E_{a}(x, 1)$ centered at $x \in \mathbb{R}^{N}$ with semi-axes $a_{1}, \ldots, a_{N}$.

Corollary 3.4. Let $\Omega$ be a domain in $\mathbb{R}^{N}, N \geq 2$, with compact boundary $\partial \Omega$ of class $C^{0}$.

If $\Omega$ satisfies (1.4) with $\Gamma=\partial \Omega$ and $B(x, r)$ replaced by $E_{a}(x, r)$, then $\partial \Omega$ must be the boundary of an ellipsoid $E_{a}(\bar{x}, \bar{r})$ for some $\bar{x} \in \mathbb{R}^{N}$ and $\bar{r}>0$.

Proof. We can always suppose that the axes of $E_{a}(x, 1)$ are parallel to the coordinate axes. Let $A$ be the $N \times N$ diagonal matrix with entries $a_{1}, \ldots, a_{N}$ on the diagonal; we have

$$
\left|\Omega \cap E_{a}(x, r)\right|=\left|A^{-1}(\Omega) \cap B\left(A^{-1}(x), r\right)\right| \cdot \operatorname{det}(A) .
$$

Moreover, since $x \in \partial \Omega$, we have $A^{-1}(x) \in \partial A^{-1}(\Omega)$. Corollary 3.3 then implies that $\partial A^{-1}(\Omega)$ is a sphere $\partial B(\bar{x}, \bar{r})$ and hence $\partial \Omega=A(\partial B(\bar{x}, \bar{r}))=\partial E_{a}(\bar{x}, \bar{r})$.

Theorem 1.3 is a consequence of the following result.

Theorem 3.5. Let $\Omega$ be a domain in $\mathbb{R}^{N}, N \geq 3$, and suppose $\Gamma$ is an open subset of the boundary $\partial \Omega$ which is of class $C^{0}$. If $\Omega$ is uniformly dense in $\Gamma$, then

$$
\rho(x, r)=\frac{1}{2}+\frac{N}{N+1} \sigma_{1}(x) r+\frac{N}{N+3} \sigma_{3}(x) r^{3}+O\left(r^{5}\right) \text { as } r \rightarrow 0, x \in \Gamma,
$$

where $\sigma_{1}(x)$, given by (3.4), and

$$
\sigma_{3}(x)= \begin{cases}\frac{1}{256}\left[K_{1}(x)^{3}-4 K_{1}(x) K_{2}(x)\right] & \text { if } N=3, \\ \frac{\omega_{N-1}}{16 \omega_{N}\left(N^{2}-1\right)}\left[K_{1}(x)^{3}-4 K_{1}(x) K_{2}(x)+4 K_{3}(x)\right] & \text { if } N \geq 4,\end{cases}
$$

are constant on $\Gamma$. 
Proof. We begin by computing $\sigma_{3}(x)$ in terms of the symmetric invariants of the surface $\Gamma$. Without loss of generality, we can suppose that $x=0$ and that $\Gamma$ is represented by the equation $y_{N}=\varphi(y)$, where $\varphi: \mathbb{R}^{N-1} \rightarrow \mathbb{R}$ is analytic and $\varphi(0)=0, \nabla \varphi(0)=0$.

In view of (5.7) in Theorem 5.2, we calculate the integrals $\int_{\mathbb{S}^{N-2}} P_{2}(v)^{3} d S_{v}$ and $\int_{\mathbb{S}^{N-2}} P_{4}(v) d S_{v}$. The bilinear form $P_{2}(v)$ can be written as

$$
P_{2}(v)=\frac{1}{2}\left\langle\nabla^{2} \varphi(0) v, v\right\rangle,
$$

where $\nabla^{2} \varphi$ denotes the Hessian matrix of $\varphi$. We choose a matrix $U \in O(N-1)$ such that $-U^{t}\left(\nabla^{2} \varphi(0)\right) U=\operatorname{diag}\left(\kappa_{1}, \ldots, \kappa_{N-1}\right)$, where the $\kappa_{j}$ 's are the eigenvalues of $-\nabla^{2} \varphi(0)$. Note that the $\kappa_{j}$ 's are nothing else than the principal curvatures of $\partial \Omega$ at $x$.

By using the change of variables $v=U \omega$, we obtain

$$
\begin{aligned}
\int_{\mathbb{S}^{N-2}} P_{2}(v)^{3} d S_{v} & =\int_{\mathbb{S}^{N-2}} P_{2}(U \omega)^{3} d S_{\omega}=-\frac{1}{8} \int_{\mathbb{S}^{N-2}}\left[\sum_{j=1}^{N-1} \kappa_{j} \omega_{j}^{2}\right]^{3} d S_{\omega} \\
& =-\frac{1}{8} \int_{\mathbb{S}^{N-2}} \sum_{|i|=3} \frac{|i| !}{i !} \kappa^{i} \omega^{2 i} d S_{\omega},
\end{aligned}
$$

where we applied the multinomial formula to the last equality. Here, as usual, we mean $\kappa^{i}=\kappa_{1}^{i_{1}} \cdots \kappa_{N-1}^{i_{N-1}}$. Thus, Lemma 5.4 yields

$$
\int_{\mathbb{S}^{N-2}} P_{2}(v)^{3} d S_{v}=-\frac{3 \omega_{N-1}}{2^{5}(N+3)\left(N^{2}-1\right)} \sum_{|i|=3} \frac{(2 i) !}{(i !)^{2}} \kappa^{i} .
$$

Lemma 5.4 also implies that

$$
\int_{\mathbb{S}^{N-2}} P_{4}(v) d S_{v}=\frac{\omega_{N-1}}{4\left(N^{2}-1\right)} \sum_{|i|=2} \frac{D^{2 i} \varphi(0)}{i !}=\frac{\omega_{N-1}}{8\left(N^{2}-1\right)} \Delta^{2} \varphi(0) .
$$

Let us first consider the case where $N \geq 4$. Observe that

$$
\frac{1}{4} \sum_{|i|=3} \frac{(2 i) !}{(i !)^{2}} \kappa^{i}=5 K_{1}^{3}-12 K_{1} K_{2}+8 K_{3} \text {; }
$$

indeed,

$$
\begin{gathered}
\sum_{j \neq h} \kappa_{j} \kappa_{h}^{2}=K_{1} K_{2}-3 K_{3}, \\
\sum_{j=1}^{N-1} \kappa_{j}^{3}=K_{1}^{3}-3 K_{1} K_{2}+3 K_{3}, \\
\sum_{|i|=3} \frac{(2 i) !}{(i !)^{2}} \kappa^{i}=20 \sum_{j=1}^{N-1} \kappa_{j}^{3}+12 \sum_{j \neq h} \kappa_{j} \kappa_{h}^{2}+8 K_{3} .
\end{gathered}
$$

Thus, from (3.6) we obtain the formula: for $N \geq 4$,

$$
\int_{\mathbb{S}^{N-2}} P_{2}(v)^{3} d S_{v}=-\frac{3 \omega_{N-1}}{8(N+3)\left(N^{2}-1\right)}\left[5 K_{1}^{3}-12 K_{1} K_{2}+8 K_{3}\right] .
$$


Since the mean curvature $H$ of $\partial \Omega$ is a constant $H_{0}$ in $\Gamma$, we have that

$$
\left(1+|\nabla \varphi|^{2}\right) \Delta \varphi-\left\langle\nabla^{2} \varphi \nabla \varphi, \nabla \varphi\right\rangle=-(N-1) H_{0}\left(1+|\nabla \varphi|^{2}\right)^{3 / 2}
$$

for any $y$ in a neighborhood of the origin 0 in $\mathbb{R}^{N-1}$. Differentiating twice this formula with respect to $y$ and using $\nabla \varphi(0)=0$ gives

$$
\Delta^{2} \varphi=-2 \Delta \varphi \operatorname{tr}\left[\left(\nabla^{2} \varphi\right)^{2}\right]+2 \operatorname{tr}\left[\left(\nabla^{2} \varphi\right)^{3}\right]-3(N-1) H_{0} \operatorname{tr}\left[\left(\nabla^{2} \varphi\right)^{2}\right]
$$

at 0 in $\mathbb{R}^{N-1}$. By simple manipulations, this formula can be recast at 0 as

$$
-\Delta^{2} \varphi=3 \sum_{j=1}^{N-1} \kappa_{j}^{3}+\sum_{j \neq h} \kappa_{j} \kappa_{h}^{2}=3 K_{1}^{3}-8 K_{1} K_{2}+6 K_{3},
$$

if we note that $-(N-1) H_{0}=\Delta \varphi(0)$.

Thus, we can write from (3.7) that

$$
\int_{\mathbb{S}^{N-2}} P_{4}(v) d S_{v}=-\frac{\omega_{N-1}}{8\left(N^{2}-1\right)}\left[3 K_{1}^{3}-8 K_{1} K_{2}+6 K_{3}\right] .
$$

Inserting formulas (3.8) and (3.9) into the third formula in (5.7) yields (3.5).

When $N=3$, we see that only the terms containing $K_{3}$ disappear in the previous calculations. Instead of (3.8) and (3.9), we have for $N=3$,

$$
\int_{\mathbb{S}^{1}} P_{2}(v)^{3} d S_{v}=-\frac{\pi}{64}\left[5 K_{1}^{3}-12 K_{1} K_{2}\right]
$$

and

$$
\int_{\mathbb{S}^{1}} P_{4}(v) d S_{v}=-\frac{\pi}{32}\left[3 K_{1}^{3}-8 K_{1} K_{2}\right],
$$

respectively. Thus, inserting formulas (3.10) and (3.11) into (5.7) yields (3.5).

Proof of Theorem 1.3. Since $\rho(x, r)$ is constant for $x \in \Gamma$ and fixed $r$, we have that $\sigma_{1}(x)$ and $\sigma_{3}(x)$ are constant on $\Gamma$, that is, assertions (i) and (ii) hold.

When $N=3$, since $\sigma_{1}$ and $\sigma_{3}$ are constant on $\Gamma$, then either $H \equiv 0$ or $H \neq 0$ and $K_{2}$ is constant on $\Gamma$. In the latter case both principal curvatures of $\Gamma$ must be constant, that is, $\Gamma$ is either a portion of a sphere or of a circular cylinder.

We now consider the general case: we assume that $\Gamma$ is any stationary isothermic surface of the solution $u$ of (1.1)-(1.2). Without loss of generality, we can suppose that $\Gamma$ lies outside $\Omega$; moreover, since by Theorem $1.1 \Omega$ is uniformly dense in $\Gamma$, we infer that $\Gamma$ must be parallel to a portion of $\partial \Omega$ at some distance $R>0$.

The following theorem is a companion to Theorem 1.2 .

Theorem 3.6. Let $\Omega$ be a domain in $\mathbb{R}^{N}, N \geq 2$, satisfying the interior sphere condition and suppose that $D$ is a domain satisfying the interior cone condition and such that $\bar{D} \subset \mathbb{R}^{N} \backslash \bar{\Omega}$. Let $\Gamma=\partial D$ and let $R=\operatorname{dist}(\Gamma, \partial \Omega)$.

If $\Omega$ is uniformly dense in $\Gamma$ (with $r_{0}$ either finite or infinite), then there exists an open subset $\Lambda$ of $\partial \Omega$, which is analytic and parallel to $\Gamma$, satisfying

$$
\prod_{i=1}^{N-1}\left[1-R \kappa_{i}(x)\right]=c \text { for every } x \in \Lambda,
$$

for some positive constant $c$. In particular, if $\partial \Omega$ is bounded and connected, $\Omega$ must be either a ball or the exterior of a ball. 
Proof. The proof parallels that of Theorem 1.2, instead of Theorem 5.2 , Theorem 5.5 must be used.

Once (3.12) is obtained, with the aid of Aleksandrov's sphere theorem (see [Al]), we can proceed as in MS1, Theorem 1.1] to obtain the spherical symmetry of $\partial \Omega$.

\section{Minimal surfaces and uniformly dense domains in $\mathbb{R}^{3}$}

Theorem 1.4 is obtained as a corollary of the following theorem.

Theorem 4.1. Let $S$ be an embedded minimal surface in $\mathbb{R}^{3}$ and let $\Omega \subset \mathbb{R}^{3}$ be a domain with $S \subset \partial \Omega$. Let $K(p)$ denote the Gauss curvature of $S$ at $p \in S$.

Suppose that there exist a sequence of points $p_{j}, j=1,2, \ldots$, in $S$ and a number $r *>0$ such that

(i) $B\left(p_{j}, r *\right) \cap S$ is represented by a graph of the function $u_{j}$ over $T \cap B\left(p_{j}, r *\right)$, where $T$ is the tangent plane of $S$ at $p_{j} \in S$;

(ii) $\sup _{p \in B\left(p_{j}, r *\right) \cap S}|K(p)| \rightarrow 0$ as $j \rightarrow+\infty$.

If $\Omega$ is uniformly dense in $S$, then $\rho(x, r)=1 / 2$ for every $x \in S$ and $r \in\left(0, r_{0}\right)$; hence $S$ must be either a portion of a plane or of a helicoid.

Proof. Let us preliminarily observe that we can always assume that $p_{j}=0, u_{j}(0)=$ 0 and $\nabla u_{j}(0)=0$, for every $j=1,2, \ldots$.

First, we shall prove that our assumptions imply that, for every non-negative integer $k$, there exists a constant $C_{k}$ such that

$$
\sup _{\sqrt{x_{1}^{2}+x_{2}^{2}} \leq \frac{1}{2} r *}\left|D^{\beta} u_{j}\left(x_{1}, x_{2}\right)\right| \leq \frac{C_{k}}{R_{j}}
$$

for any multi-index $\beta$ with $|\beta|=k$ and any $j=1,2, \ldots$.

In fact, $\kappa_{i}(p)(i=1,2)$ denote the principal curvatures of $S$ at $p$; since $S$ is a minimal surface, from (ii) we have that

$$
\max _{i=1,2} \sup _{p \in B\left(p_{j}, r *\right) \cap S}\left|\kappa_{i}(p)\right| \rightarrow 0 \text { as } j \rightarrow+\infty .
$$

Therefore, there exists a diverging sequence of positive numbers $\left\{R_{j}\right\}$ such that each set $B\left(p_{j}, r *\right) \cap S$ is contained between two balls with radius $R_{j}$ touching at $p_{j}$ (on opposite sides of $S$ ). This fact yields that there exists a constant $A>0$ satisfying

$$
\sup _{\sqrt{x_{1}^{2}+x_{2}^{2}} \leq \frac{3}{4} r *}\left|u_{j}\left(x_{1}, x_{2}\right)\right| \leq \frac{A}{R_{j}}, \quad j=1,2, \ldots
$$

We now use the a priori interior estimates for the minimal surface equation (see GT, Corollary 16.7, p. 407]): we see that, for any non-negative integer $k$, there exists a constant $A_{k}$ such that

$$
\sup _{\sqrt{x_{1}^{2}+x_{2}^{2}} \leq \frac{2}{3} r *}\left|D^{\beta} u_{j}\left(x_{1}, x_{2}\right)\right| \leq A_{k}
$$

for any multi-index $\beta$ with $|\beta|=k$ and any $j=1,2, \ldots$ With the aid of (4.3) and Schauder's interior estimates for higher order derivatives (see GT, Problem 6.1. 
(a), p. 141]), we obtain that, for any nonnegative integer $k$, there exists a constant $\tilde{A}_{k}$ such that the inequality

$$
\sup _{\sqrt{x_{1}^{2}+x_{2}^{2}} \leq \frac{1}{2} r *}\left|D^{\beta} u_{j}\left(x_{1}, x_{2}\right)\right| \leq \tilde{A}_{k} \sup _{\sqrt{x_{1}^{2}+x_{2}^{2}} \leq \frac{2}{3} r *}\left|u_{j}\left(x_{1}, x_{2}\right)\right|
$$

holds for any multi-index $\beta$ with $|\beta|=k$ and any $j=1,2, \ldots$ This inequality and (4.2) give (4.1).

Second, we use the series expansions (5.9) for each $\sigma\left(p_{j}, r\right)$ or $\rho\left(p_{j}, r\right)$ and Remark 5.3 to infer that $\rho(x, r)$ or $\sigma(x, r)$ equals $\frac{1}{2}$ for every $x \in S$ and every $r \in\left(0, r_{0}\right)$.

Since, by Remark [5.3, each coefficient $\sigma_{n}\left(p_{j}\right), n=1,2, \ldots$, is a polynomial of the variables $D^{\beta} u_{j}(0,0), 2 \leq|\beta| \leq n+1$, without zeroth order coefficient, (4.1) implies that

$$
\sigma_{n}\left(p_{j}\right) \rightarrow 0 \text { as } j \rightarrow+\infty
$$

for each $n=1,2, \ldots$. The fact that $\Omega$ is uniformly dense in $S$ and (4.4) yield that every $\sigma_{n}(x)$ must vanish for every $x \in S$ and hence $\sigma(x, r)=\rho(x, r)=1 / 2$ for every $x \in S$ and for every $r \in\left(0, r_{0}\right)$.

Finally, by Nitsche's result [N], we can conclude that $S$ must be either a portion of a plane or of a helicoid.

Proof of Theorem 1.4. We recall from [PR, p. 18] that "a complete embedded minimal surface in $\mathbb{R}^{3}$ with finite total curvature, outside a big ball in space, has a nice shape: there are a finite number of parallel ends and each end is asymptotic to a plane or to a halfcatenoid" (see also [HK, Proposition 2.5, pp. 36 - 37] for a more precise description concerning complete, nonplanar, minimal surfaces with finite total curvature).

As in $\mathrm{PR}$, we may assume that every end of $S$ is horizontal. The maximum principle at infinity (see $[\mathrm{LR}$ ) guarantees that every connected component of $S$ outside a big ball has a positive distance from the other components - each component can be represented by a graph of a function $u=u\left(x_{1}, x_{2}\right)$ over the exterior of the big disk in $\mathbb{R}^{2}$. Also, it is shown in $[\mathbf{S}]$ that each function $u$ has the following asymptotic behavior for $|x|\left(=\sqrt{x_{1}^{2}+x_{2}^{2}}\right)$ large:

$$
u(x)=a \log |x|+b+\frac{c_{1} x_{1}}{|x|^{2}}+\frac{c_{2} x_{2}}{|x|^{2}}+O\left(|x|^{-2}\right)
$$

for some constants $a, b, c_{1}, c_{2}$.

Therefore, as in [S, Proposition 1, p. 801], the Gauss curvature $K(p)$ of $S$ at $p=(x, u(x))$ satisfies

$$
|K(p)|=O\left(|x|^{-4}\right)
$$

for large $|x|$.

Hence, by choosing a sequence of points $p_{j}, j=1,2, \ldots$, in $S$ in Theorem 4.1 such that

$$
p_{j}=\left(x^{j}, u\left(x^{j}\right)\right) \text { and }\left|x^{j}\right|=\sqrt{\left(x_{1}^{j}\right)^{2}+\left(x_{2}^{j}\right)^{2}} \rightarrow+\infty \text { as } j \rightarrow+\infty,
$$

we see that Theorem 4.1 implies Theorem 1.4 . 


\section{TAYLOR FORMULAS FOR $\rho(x, r)$ AND $\sigma(x, r)$}

The Taylor expansion for $\sigma(x, r)$ and $\rho(x, r)$ at $r=0$ will be computed in Theorem 5.2. The proof of this theorem is based on Lemma 5.1, where we will compute the coefficients of the Taylor expansion at $r=0$ for the function $\sigma(x, r)$ defined in (2.2) in terms of a particular parameterization of the surface $\partial \Omega$ in a neighborhood of $x$.

Suppose that $\partial \Omega$ is smooth and, for $x \in \partial \Omega$, denote by $T_{x}(\partial \Omega)$ and $\nu$ the tangent space and the normal unit vector to $\partial \Omega$ at $x$, respectively. For fixed $v \in T_{x}(\partial \Omega)$ with $|v|=1$, let $\pi_{x}(v, \nu)$ be the plane through $x$ spanned by $v$ and $\nu$. In Lemma 5.1. we assume that, for $r>0$ sufficiently small, each point $z$ in $\Omega \cap B(x, r)$ can be parameterized in spherical coordinates as

$$
\begin{aligned}
& z=x+\rho \cos \phi v+\rho \sin \phi \nu, \\
& v \in T_{x}(\partial \Omega) \cap \mathbb{S}^{N-2}, \theta(\rho, v) \leq \phi \leq \pi / 2,0 \leq \rho \leq r,
\end{aligned}
$$

where, for fixed $v \in T_{x}(\partial \Omega) \cap \mathbb{S}^{N-2}, \theta=\theta(\rho, v)$ parameterizes the curve $\partial \Omega \cap \pi_{x}(v, \nu)$ in polar coordinates.

Lemma 5.1. Suppose that $\partial \Omega$ is analytic in a neighborhood of a point $x \in \partial \Omega$ and let the function $\theta(\rho, v)$ parameterize $\partial \Omega$ as specified in (5.1). Then the functions $\sigma(x, r)$ and $\rho(x, r)$, defined in (2.2) and (1.3), respectively, admit the following Taylor formulas:

$$
\begin{aligned}
& \sigma(x, r)=\frac{1}{2}+\sigma_{1}(x) r+\sigma_{2}(x) r^{2}+\sigma_{3}(x) r^{3}+O\left(r^{4}\right), \\
& \rho(x, r)=\frac{1}{2}+\frac{N}{N+1} \sigma_{1}(x) r+\frac{N}{N+2} \sigma_{2}(x) r^{2}+\frac{N}{N+3} \sigma_{3}(x) r^{3}+O\left(r^{4}\right)
\end{aligned}
$$

as $r \rightarrow 0$, where

$$
\begin{aligned}
& \sigma_{1}(x)=-\frac{1}{\omega_{N}} \int_{\mathbb{S}^{N-2}} \theta_{1}(v) d S_{v}, \quad \sigma_{2}(x)=-\frac{1}{\omega_{N}} \int_{\mathbb{S}^{N-2}} \theta_{2}(v) d S_{v}, \\
& \sigma_{3}(x)=-\frac{1}{\omega_{N}} \int_{\mathbb{S}^{N-2}}\left[\theta_{3}(v)-\frac{N-2}{6} \theta_{1}(v)^{3}\right] d S_{v},
\end{aligned}
$$

and we have set

$$
\theta(r, v)=\theta_{1}(v) r+\theta_{2}(v) r^{2}+\theta_{3}(v) r^{3}+\cdots .
$$

Proof. The Jacobian of the change of variables (5.1) is $\rho^{N-1} \cos ^{N-2} \phi$, so that we can write

$$
|\Omega \cap B(x, r)|=\int_{\mathbb{S}^{N-2}} \int_{0}^{r} \rho^{N-1} \int_{\theta(\rho, v)}^{\pi / 2} \cos ^{N-2} \phi d \phi d \rho d S_{v},
$$

where $d S_{v}$ denotes the surface element on $\mathbb{S}^{N-2}$. By differentiating this formula with respect to $r$ and dividing by $\omega_{N} r^{N-1}$, we get

$$
\sigma(x, r)=\frac{1}{\omega_{N}} \int_{\mathbb{S}^{N-2}} \int_{\theta(r, v)}^{\pi / 2} \cos ^{N-2} \phi d \phi d S_{v}=\frac{1}{2}-\frac{1}{\omega_{N}} \int_{\mathbb{S}^{N-2}} \int_{0}^{\theta(r, v)} \cos ^{N-2} \phi d \phi d S_{v} .
$$


The first formula in (5.2) is then derived at once by observing that

$$
\int_{0}^{\theta(r, v)} \cos ^{N-2} \phi d \phi=\theta_{1}(v) r+\theta_{2}(v) r^{2}+\left[\theta_{3}(v)-\frac{N-2}{6} \theta_{1}(v)^{3}\right] r^{3}+O\left(r^{4}\right) .
$$

The second formula in (5.2) easily follows from the first one.

In Theorem 5.2 below, we suppose that $\partial \Omega$ is the graph of an analytic function $\varphi$ in a neighborhood of a point $x \in \partial \Omega$ and we compute the coefficients (5.4) in terms of the derivatives of $\varphi$. In particular, hereafter we will use the letter $y$ to denote an element of $\mathbb{R}^{N-1}$, that is, $y=\left(y_{1}, \ldots, y_{N-1}\right) \in \mathbb{R}^{N-1}$. Without loss of generality, we will suppose that $x$ is the origin in $\mathbb{R}^{N}$, and $T_{x}(\partial \Omega)$ coincides with the hyperplane $\left\{\left(y, y_{N}\right) \in \mathbb{R}^{N}: y_{N}=0\right\}$. The function $\varphi: \mathbb{R}^{N-1} \rightarrow \mathbb{R}$ then parameterizes $\partial \Omega$ in a neighborhood of $x=0$, that is, $\partial \Omega$ is represented by the equation $y_{N}=\varphi(y)$, where $\varphi(0)=0$ and $\nabla \varphi(0)=0$.

We will also use a standard multi-index notation for the derivatives of $\varphi$ : if $i=\left(i_{1}, \ldots, i_{N-1}\right)$ is a multi-index, we denote $|i|=i_{1}+\cdots+i_{N-1}, i !=i_{1} ! \cdots i_{N-1} !$,

$$
D^{i} \varphi=\partial_{y_{1}}^{i_{1}} \cdots \partial_{y_{N-1}}^{i_{N-1}} \varphi
$$

and $y^{i}=y_{1}^{i_{1}} \cdots y_{N-1}^{i_{N-1}}$ for $y \in \mathbb{R}^{N-1}$. With these notations and assumptions, the Taylor expansion of $\varphi$ in a neighborhood of $y=0$ is

$$
\varphi(y)=\sum_{n=2}^{\infty} P_{n}(y), \text { where } P_{n}(y)=\sum_{|i|=n} \frac{D^{i} \varphi(0)}{i !} y^{i}, n=0,1, \ldots
$$

Theorem 5.2. Let $\partial \Omega$ be analytic in a neighborhood of a point $x \in \partial \Omega$.

Then the functions $\sigma(x, r)$ and $\rho(x, r)$ defined in (2.2) and (1.3), respectively, admit the Taylor expansions (5.2), where

$$
\begin{aligned}
& \sigma_{1}(x)=-\frac{1}{\omega_{N}} \int_{\mathbb{S}^{N-2}} P_{2}(v) d S_{v}, \quad \sigma_{2}(x)=-\frac{1}{\omega_{N}} \int_{\mathbb{S}^{N-2}} P_{3}(v) d S_{v}, \\
& \sigma_{3}(x)=-\frac{1}{\omega_{N}} \int_{\mathbb{S}^{N-2}}\left[P_{4}(v)-\frac{N+3}{6} P_{2}(v)^{3}\right] d S_{v} .
\end{aligned}
$$

Proof. We just have to compute the integrands in (5.3) in terms of the polynomials $P_{n}(v)$ defined in (5.6).

From (5.6) we write

$$
\varphi(r \cos \theta(r, v) v)=\sum_{n=2}^{\infty} r^{n} \cos ^{n} \theta(r, v) P_{n}(v) .
$$

Since $r \sin \theta(r, v)=\varphi(r \cos \theta(r, v) v)$ for sufficiently small $r$, we have

$$
\sin \theta(r, v)=\sum_{n=2}^{4} r^{n-1} \cos ^{n} \theta(r, v) P_{n}(v)+O\left(r^{4}\right) .
$$

Now, we use the formulas: as $\theta \rightarrow 0$,

$$
\begin{aligned}
& \sin \theta=\theta-\frac{1}{6} \theta^{3}+O\left(\theta^{5}\right), \quad \cos ^{2} \theta=1-\theta^{2}+O\left(\theta^{4}\right), \\
& \cos ^{3} \theta=1+O\left(\theta^{2}\right), \quad \cos ^{4} \theta=1+O\left(\theta^{2}\right),
\end{aligned}
$$


and, by (5.4) and (5.8), we obtain: as $r \rightarrow 0$,

$$
\begin{aligned}
& \theta_{1}(v) r+\theta_{2}(v) r^{2}+\left[\theta_{3}(v)-\frac{1}{6} \theta_{1}(v)^{3}\right] r^{3}+O\left(r^{4}\right) \\
= & P_{2}(v) r+P_{3}(v) r^{2}+\left[P_{4}(v)-\theta_{1}(v)^{2} P_{2}(v)\right] r^{3}+O\left(r^{4}\right) .
\end{aligned}
$$

From this formula, we have

$$
\begin{aligned}
& \theta_{1}(v)=P_{2}(v), \quad \theta_{2}(v)=P_{3}(v), \\
& \theta_{3}(v)=\frac{1}{6} \theta_{1}(v)^{3}+P_{4}(v)-\theta_{1}(v)^{2} P_{2}(v)=P_{4}(v)-\frac{5}{6} P_{2}(v)^{3},
\end{aligned}
$$

and hence (5.7) follows at once.

Remark 5.3. It can be shown that $\sigma(x, r)$ and $\rho(x, r)$ admit the series expansions

$$
\sigma(x, r)=\frac{1}{2}+\sum_{n=1}^{\infty} \sigma_{n}(x) r^{n} \text { and } \rho(x, r)=\frac{1}{2}+\sum_{n=1}^{\infty} \frac{N}{N+n} \sigma_{n}(x) r^{n} .
$$

Here, for each $n \in \mathbb{N}$, the integrand in the expression for $\sigma_{n}(x)$ is a polynomial, without zeroth order coefficient, of the functions $P_{2}(v), \ldots, P_{n+1}(v)$, and hence each coefficient $\sigma_{n}(x)(n \in \mathbb{N})$ is a polynomial, without zeroth order coefficient, of $D^{\beta} \varphi(0,0), 2 \leq|\beta| \leq n+1$. In particular, we obtain that $\sigma_{2 k}(x)=0$ for any $k=1,2, \ldots$.

Lemma 5.4. Let $i=\left(i_{1}, \ldots, i_{N-1}\right)$ be a multi-index. We have that the moments

$$
\int_{\mathbb{S}^{N-2}} v^{i} d S_{v}=0
$$

if at least one entry of $i$ is odd; otherwise, when $N=2$,

$$
\frac{1}{\omega_{N-1}} \int_{\mathbb{S}^{N-2}} v^{2 i} d S_{v}=1
$$

and when $N \geq 3$,

$$
\frac{1}{\omega_{N-1}} \int_{\mathbb{S}^{N-2}} v^{2 i} d S_{v}=\frac{(N-3) ! !(2 i) !}{(2|i|+N-3) ! ! 2^{|i|} i !},
$$

where $n ! !=\prod_{k=0}^{\left[\frac{n-1}{2}\right]}(n-2 k)$.

Proof. Since the proof is easy when $N=2$, we assume $N \geq 3$. Let

$$
F_{i}=\int_{\partial B(0,1)} y^{i} d S_{y}
$$

let $e_{j}$ be the $j$-th canonical direction, and let $\nu_{j}(y)$ be the $j$-th component of the unit normal to $\partial B(0,1)$ at $y \in \partial B(0,1)$. In the following chain of equalities, we use the divergence theorem and the coarea identity: if $i_{j} \geq 2$, then

$$
\begin{aligned}
F_{i} & =\int_{\partial B(0,1)} y^{i-e_{j}} \nu_{j}(y) d S_{y}=\int_{B(0,1)} \frac{\partial}{\partial y_{j}}\left(y^{i-e_{j}}\right) d y \\
& =\left(i_{j}-1\right) \int_{B(0,1)} y^{i-2 e_{j}} d y=\left(i_{j}-1\right) \int_{0}^{1}\left(\int_{\partial B(0, r)} y^{i-2 e_{j}} d S_{y}\right) d r \\
& =\left(i_{j}-1\right) \int_{0}^{1} F_{i-2 e_{j}} r^{|i|+N-4} d r
\end{aligned}
$$


thus we obtain the recurrence relation:

$$
F_{i}=\frac{i_{j}-1}{|i|+N-3} F_{i-2 e_{j}}, i_{j} \geq 2 .
$$

Since $F_{(0, \ldots, 0)}=\omega_{N-1}$, iterating this formula easily yields the conclusion.

In the sequel, we derive an asymptotic formula for $\sigma(x, r)$ in the case where the point $x$ lies outside $\bar{\Omega}$ at a positive distance $R$ from it. Consider the case where there exists a unique point $\pi(x) \in \partial \Omega$ such that $\overline{B(x, R)} \cap \bar{\Omega}=\{\pi(x)\}$. In this case, the relevant parameter is no longer the radius $r$ but rather $r-R$.

Our starting point is the conclusions of Theorem 2.5. In particular, the conclusion (iii) will guarantee the parameterization (5.1) for $r-R>0$ sufficiently small, that is, it will guarantee the existence and uniqueness of the solution $\theta=\theta(r, v)$ of the equation $r \sin \theta=\varphi(r \cos \theta v)$ for $r-R>0$ sufficiently small and for every $v \in T_{\pi(x)}(\partial \Omega) \cap \mathbb{S}^{N-2}$. Indeed, we choose the coordinate axes $\left(y, y_{N}\right)$ with the origin at $x$ and the $y_{N}$ axis passing through the point $\pi(x)$. In this way, $\partial \Omega$ can be represented by the equation $y_{N}=\varphi(y)$, where $y \in \mathbb{R}^{N-1}$ ranges in a neighborhood of $0, \varphi(0)=R$ and $\nabla \varphi(0)=0$. By a further rotation in $y$ we may assume that $-\nabla^{2} \varphi(0)=\operatorname{diag}\left(\kappa_{1}, \ldots, \kappa_{N-1}\right)$, where $\kappa_{i}=\kappa_{i}(\pi(x))(i=1, \ldots, N-1)$ denote the principal curvatures of $\partial \Omega$ at $\pi(x) \in \partial \Omega$. In our calculations, it is convenient to introduce the non-dimensional parameter $s=(r-R) / R$ and the re-scaling

$$
\theta=\frac{\pi}{2}-\sqrt{\psi}
$$

Define the function $G=G(s, v, \psi)$ by

$$
G(s, v, \psi)=(1+s) R \cos \sqrt{\psi}-\varphi((1+s) R \sin \sqrt{\psi} v)(=r \sin \theta-\varphi(r \cos \theta v)) .
$$

Note that

$$
G(0, v, 0)=0 \text { for every } v \in \mathbb{S}^{N-2} .
$$

Observe that as $\psi \rightarrow 0$

$$
G(s, v, \psi)=(1+s) R \cos \sqrt{\psi}-R+\frac{1}{2}(1+s)^{2} R^{2} \sin ^{2} \sqrt{\psi} \sum_{i=1}^{N-1} \kappa_{i} v_{i}^{2}+O\left(\psi^{\frac{3}{2}}\right),
$$

where the remainder term $O\left(\psi^{\frac{3}{2}}\right)$ is uniform for $|s|$ bounded and for $v \in \mathbb{S}^{N-2}$. Hence $G$ extends as a $C^{1}$ function in a neighborhood of $\psi=0$, and for every $v \in \mathbb{S}^{N-2}$

$$
\frac{\partial G}{\partial \psi}(0, v, 0)=-\frac{R^{2}}{2}\left(\frac{1}{R}-\sum_{i=1}^{N-1} \kappa_{i} v_{i}^{2}\right)<0,
$$

where we have used conclusion (iii) of Theorem 2.5. that is, $\kappa_{i}<\frac{1}{R}(i=1, \ldots$, $N-1)$. Therefore, by virtue of (5.13) and (5.14), it follows from the implicit function theorem and compactness of $\mathbb{S}^{N-2}$ that there exist a unique $C^{1}$ function $\psi=\psi(s, v)$ and a number $\delta>0$ satisfying

$$
\psi(0, v)=G(s, v, \psi(s, v))=0 \text { for every }(s, v) \in(-\delta, \delta) \times \mathbb{S}^{N-2} .
$$

Moreover, observe that

$$
\frac{\partial G}{\partial s}(0, v, 0)=R>0 \text { for every } v \in \mathbb{S}^{N-2} .
$$


Hence, by combining this with (5.14) we may have

$$
\psi(s, v)>0 \text { for every }(s, v) \in(0, \delta) \times \mathbb{S}^{N-2} .
$$

Eventually, formula (5.5) still holds, and as before, we obtain that

$$
\sigma(x, r)=\frac{1}{\omega_{N}} \int_{\mathbb{S}^{N-2}} \int_{\theta(r, v)}^{\pi / 2} \cos ^{N-2} \phi d \phi d S_{v}
$$

and by setting $\tilde{\sigma}(x, s)=\sigma(x, R+R s)$ we have

$$
\tilde{\sigma}(x, s)=\frac{1}{\omega_{N}} \int_{\mathbb{S}^{N-2}} \int_{0}^{\sqrt{\psi(s, v)}} \sin ^{N-2} \phi d \phi d S_{v} .
$$

Furthermore, differentiating the second equation in 5.15 with respect to $s$ and setting $s=0$ yield

$$
\frac{\partial G}{\partial s}(0, v, 0)+\frac{\partial G}{\partial \psi}(0, v, 0) \frac{\partial \psi}{\partial s}(0, v)=0 .
$$

By combining this with (5.14) and (5.16), we conclude that

$$
\psi(s, v)=2\left[1-R \sum_{i=1}^{N-1} \kappa_{i} v_{i}^{2}\right]^{-1} s+o(s) \text { as } s \rightarrow 0^{+},
$$

where the remainder term $o(s)$ is uniform for $v \in \mathbb{S}^{N-2}$.

Theorem 5.5. Let $\Omega \subset \mathbb{R}^{N}$ be a domain with smooth boundary $\partial \Omega$, let $x \notin \bar{\Omega}$ and put $R=\operatorname{dist}(x, \partial \Omega)$. Assume that $\overline{B(x, R)} \cap \bar{\Omega}=\{\pi(x)\}$ and each principal curvature $\kappa_{i}(\pi(x))$ is smaller than $\frac{1}{R}$, where $\pi(x)$ is the projection of $x$ on $\partial \Omega$.

The following asymptotic formula holds:

$$
\tilde{\sigma}(x, s)=s^{\frac{N-1}{2}} \tilde{\sigma}_{1}(x)+o\left(s^{\frac{N-1}{2}}\right) \text { as } s \rightarrow 0^{+},
$$

where

$$
\tilde{\sigma}_{1}(x)=\frac{2^{\frac{N-1}{2}} \omega_{N-1}}{(N-1) \omega_{N} \sqrt{\prod_{i=1}^{N-1}\left[1-R \kappa_{i}(\pi(x))\right]}} .
$$

Proof. By (5.19) we get

$$
\sqrt{\psi(s, v)}=\sqrt{2}\left[1-R \sum_{i=1}^{N-1} \kappa_{i}(\pi(x)) v_{i}^{2}\right]^{-\frac{1}{2}} \sqrt{s}(1+o(1)) \text { as } s \rightarrow 0^{+},
$$

where the remainder term $o(1)$ is uniform for $v \in \mathbb{S}^{N-2}$. Therefore, since

$$
\sin ^{N-2} \phi=\phi^{N-2}+O\left(\phi^{N}\right) \text { as } \phi \rightarrow 0,
$$

formula (5.18) yields (5.20), where

$$
\tilde{\sigma}_{1}(x)=\frac{2^{\frac{N-1}{2}}}{(N-1) \omega_{N}} \int_{\mathbb{S}^{N-2}}\left[1-R \sum_{i=1}^{N-1} \kappa_{i}(\pi(x)) v_{i}^{2}\right]^{-\frac{N-1}{2}} d S_{v} .
$$


Thus formula (5.21) follows from Lemma 5.6 by observing that, if $v \in \mathbb{S}^{N-2}$, then

$$
1-R \sum_{i=1}^{N-1} \kappa_{i}(\pi(x)) v_{i}^{2}=\sum_{i=1}^{N-1}\left[1-R \kappa_{i}(\pi(x))\right] v_{i}^{2} .
$$

Lemma 5.6. Let $w_{1}, \ldots, w_{N-1}$ be positive numbers. Then we have

$$
\int_{\mathbb{S}^{N-2}}\left[\sum_{i=1}^{N-1} w_{i} v_{i}^{2}\right]^{-(N-1) / 2} d S_{v}=\omega_{N-1}\left[\prod_{i=1}^{N-1} w_{i}\right]^{-1 / 2} .
$$

Proof. Since

$$
\operatorname{div}\left\{\left[\sum_{i=1}^{N-1} w_{i} y_{i}^{2}\right]^{-\frac{N-1}{2}} y\right\}=0
$$

for $y \neq 0$, then, by the divergence theorem, the integral in (5.23) equals

$$
\int_{\left\{v: \sum_{i=1}^{N-1} w_{i} v_{i}^{2}=1\right\}}\left[\sum_{i=1}^{N-1} w_{i} v_{i}^{2}\right]^{-(N-1) / 2} v \cdot \nu(v) d S_{v}=\int_{\left\{v: \sum_{i=1}^{N-1} w_{i} v_{i}^{2}=1\right\}} v \cdot \nu(v) d S_{v} .
$$

By the divergence theorem, the last integral equals $N-1$ times the volume of the ellipsoid $\left\{y: \sum_{i=1}^{N-1} w_{i} y_{i}^{2} \leq 1\right\}$.

\section{ACKNOWLEDGEMENT}

The authors are indebted to Professor Makoto Kimura who suggested the parameterization (5.1). Corollary 3.4 was inspired by a remark of Professor Roberto Gianni.

The main results of the paper were discovered while R. Magnanini and J. Prajapat were visiting the Department of Mathematics of Ehime University; they wish to thank that department for its kind hospitality.

\section{REFERENCES}

[Al] A. D. Aleksandrov, Uniqueness theorems for surfaces in the large V, Vestnik Leningrad Univ. 13, no. 19 (1958), 5-8. (English translation: Amer. Math. Soc. Translations, Ser. 2, 21 (1962), 412-415.) MR0102114 (21:909)

[As] G. Alessandrini, Matzoh ball soup: a symmetry result for the heat equation, J. Analyse Math. 54 (1990), 229-236. MR1041182 (91d:31001)

[CS] M. Chamberland and D. Siegel, Convex domains with stationary hot spots, Math. Meth. Appl. Sci. 20 (1997), 1163-1169. MR1468407 (98g:35083)

[CK] I. Chavel and L. Karp, Movement of hot spots in Riemannian manifolds, J. Analyse Math. 55 (1990), 271-286. MR.1094719 (92b:58223)

[GT] D. Gilbarg and N. S. Trudinger, Elliptic Partial Differential Equations of Second Order, (Second Edition), Springer-Verlag, Berlin, Heidelberg, New York, Tokyo, 1983. MR0737190 (86c:35035)

[GW] R. Gulliver and N. B. Willms, A conjectured heat flow problem, in Solutions, SIAM Review 37 (1995), 100-104.

[HK] D. Hoffman and H. Karcher, Complete embedded minimal surfaces of finite total curvature, in Geometry V, Encyclopaedia of Math. Sci. 90 (R. Osserman, ed.), Springer-Verlag, Berlin, Heidelberg, New York, 1997, 5-93. MR1490038 (98m:53012) 
[Kp] N. Kapouleas, Complete embedded minimal surfaces of finite total curvature, J. Differential Geometry 45 (1997), 95-169. MR.1601434 (99a:53008)

[Kw] B. Kawohl, A conjectured heat flow problem, in Solutions, SIAM Review 37 (1995), 104105.

[Kl] M. S. Klamkin, A conjectured heat flow problem, in Problems, SIAM Review 36 (1994), 107.

[LR] R. Langevin and H. Rosenberg, A maximum principle at infinity for minimal surfaces and applications, Duke Math. J. 57 (1988), 819-828. MR0975123 (90c:53025)

[LM] F. J. López and F. Martín, Complete minimal surfaces in $\mathbb{R}^{3}$, Publicacions Matemàtiques 43 (1999), 341-449. MR:1744617(2002c:53010)

[Ma] R. Magnanini, On symmetric invariants of level surfaces near regular points, Bull. London Math. Soc. 24 (1992), 565-574. MR.1183313 (93i:53055)

[MS1] R. Magnanini and S. Sakaguchi, Matzoh ball soup: heat conductors with a stationary isothermic surface, Ann. of Math. 156 (2002), 931-946. MR.1954240 (2003k:35086)

[MS2] R. Magnanini and S. Sakaguchi, On heat conductors with a stationary hot spot, Ann. Mat. Pura Appl. 183 (2004), 1-23. MR2044330 (2005b:35104)

[MS3] R. Magnanini and S. Sakaguchi, Stationary isothermic surfaces for unbounded domains, in preparation.

[MS4] R. Magnanini and S. Sakaguchi, The spatial critical points not moving along the heat flow, J. Analyse Math. 71 (1997), 237-261. MR1454253(99e:35087)

[N] J. C. C. Nitsche, Characterizations of the mean curvature and a problem of G. Cimmino, Analysis 15 (1995), 233-245. MR1357961 (97c:53009)

[PR] J. Pérez and A. Ros, Properly embedded minimal surfaces with finite total curvature, Lecture Notes in Math. 1775, Springer-Verlag, Berlin, Heidelberg, New York, Tokyo, 2002, 15-66. MR.1901613

[R] R. C. Reilly, On the Hessian of a function and the curvatures of its graph, Michigan Math. J. 20 (1973), 373-383. MR0334045 (48:12364)

[S] R. Schoen, Uniqueness, symmetry, and embeddedness of minimal surfaces, J. Differential Geometry 18 (1983), 791-809. MR0730928 (85f:53011)

[T] M. Traizet, An embedded minimal surface with no symmetries, J. Differential Geometry 60 (2002), 103-153. MR1924593 (2004c:53008)

[Z] L. Zalcman, Some inverse problems of potential theory, Contemp. Math. 63 (1987), 337350. MR0876329 (88e:31012)

Dipartimento di Matematica U. Dini, Università di Firenze, viale Morgagni 67/A, 50134 FiREnZe, ItALy

E-mail address: magnanin@math.unifi.it

Indian Statistical Institute, Stat-Math Unit, 8th Mile, Mysore Road, R.V.C.E. Post, BANGALORE 560 059, INDIA

E-mail address: jyotsna@isibang.ac.in

Current address: Department of Mathematics, Indian Institute of Technology Bombay, Powai, Mumbai 400 097, India

Department of Mathematics, Faculty of Science, Ehime University, 2-5 Bunkyo-cho, MATSUYAMA-SHI, EHIME 790-8577 JAPAN

E-mail address: sakaguch@dpc.ehime-u.ac.jp 